\title{
FORMES, FONCTIONS ET CATÉGORIES LINGUISTIQUES: LES PRINCIPES DE GRAMMAIRE GÉNÉRALE DE LOUIS HJELMSLEV
}

Quand, en 1928, Louis Hjelmslev publie ses Principes de grammaire générale, i1 s'inscrit dans un vaste processus d'élaboration d'une linguistique générale, qui permet de rattacher ces prémices d'une œuvre ${ }^{1}$ qu'on associe aujourd'hui avec une école structuraliste spécifique - la glossématique -, aux travaux fondateurs de Saussure, de Jespersen ${ }^{2}$, de Sapir. On pourrait parler de "insertion dans l'histoire des théories linguistiques", car, comme Saussure, Hjelmslev a senti la nécessité de formuler, pour la première fois, une théorie cohérente du langage et de repartir de zéro, tout en acceptant les acquis "matériels" du passé.

"Il est donc impossible de tracer le développement de la théorie du langage et d'en écrire l'histoire: il lui manque la continuité. À cause de cela, tout effort pour formuler une théorie du langage s'est vu discrédité et considéré comme une vaine philosophie, un dilettantisme teinté d'apriorisme. La condamnation semble d'ailleurs justifiée, car dans ce domaine, dilettantisme et apriorisme ont prévalu à tel point que, du dehors, il est souvent difficile de distinguer le vrai du faux. Le présent ouvrage voudrait contribuer à faire reconnaître que de telles caractéristiques ne sont pas nécessairement inhérentes à toute tentative de fonder une théorie du langage. Il sera plus aisé d'y parvenir si l'on s'efforce d'oublier le passé et de faire table rase partout où il n'a rien fourni de positif pouvant être utilisé. Dans une grande mesure nous nous appuierons sur les matériaux recueillis par la recherche linguistique antérieure, matériaux, qui, réinterprétés, constitueront l'essentiel de la théorie du langage. Nous adhérons explicitement au passé sur certains points où nous savons que des résultats positifs ont été atteints par d'autres avant nous" (1943 [trad. française de 1971: 13-14]) ${ }^{3}$.

Le projet hjelmslévien de fondation d'une théorie du langage n'est pas sans rappeler les ambitions de Ferdinand de Saussure ${ }^{4}$. En 1894, dans une lettre à Antoine

\footnotetext{
1 Pour une vue d'ensemble de cette œuvre, voir Albrecht (1988: 61-66, 121-129).

2 Hjelmslev a lu attentivement tous les ouvrages importants de Jespersen: Studier over engelske kasus (1891), Growth and Structure of the English Language (1905), Sprogets logik (1913), Language: Its nature, development and origin (1921), Philosophy of Grammar (1924), Sprogets udvikling og opståen (1926), A Modern English Grammar on Historical Principles (1909-1949).

${ }^{3} \mathrm{Au}$ lieu de citer le texte original (Hjelmslev 1943: 8-9), nous citons la deuxième traduction française (celle de 1971, et non la première de 1968) de l'Omkring sprogteoriens grundlceggelse. Les renvois sont aux pages de la traduction française. Pour une analyse globale de l'Omkring sprogteoriens grundloggelse, voir FischerJørgensen (1943) et Swiggers (1995).

${ }^{4}$ Cf. Hjelmslev (1943 [trad. française de 1971: 14]): "Un seul théoricien mérite d'être cité comme un devancier indiscutable: le Suisse Ferdinand de Saussure".
} 
Meillet, Saussure formulait le constat que la plupart des linguistes "n'ont même pas la velléité de s'élever à ce degré d'abstraction qui est nécessaire pour dominer d'une part ce qu'on fait, d'autre part en quoi ce qu'on fait a une légitimité et une raison d'être dans l'ensemble des sciences". C'est que le champ de la linguistique avait été défini en fonction d'un certain type de travail, et non en fonction du rapport qui existe entre un objet et une visée, un point de vue. Placé devant l'obligation d'enseigner un cours de linguistique générale, Saussure ne pouvait que relever l'état d'indigence théorique où se trouvait la science du langage. Le parcours historique dressé au début du Cours, ainsi que les notes sur Whitney, et d'autres notes manuscrites datant de la période 1891-1911, montrent que les trois phases dans l'évolution de la linguistique - la phase de la grammaire normative, la phase de la philologie, et celle de la grammaire historico-comparative - sont caractérisées par un fait négatif: celui de ne pas avoir constitué "la véritable science linguistique", faute d'avoir dégagé la nature de son objet d'étude 5 .

Du début des années 1890 jusqu'à sa mort, Saussure s'efforcera de mettre en place un système de linguistique générale. Il s'agit pour lui d'ordonner logiquement des concepts, de mettre en place un système hiérarchique de principes et d'options théoriques:

"Mais je suis bien dégoûté de tout cela et de la difficulté qu'il y a en général à écrire seulement dix lignes ayant le sens commun en matière de faits de langage. Préoccupé surtout depuis longtemps de la classification logique de ces faits, de la classification des points de vue sous lesquels nous les traitons, je vois de plus en plus à la fois l'immensité du travail qu'il faudrait pour montrer au linguiste ce qu'il fait, en réduisant chaque opération à sa catégorie prévue, et en même temps l'assez grande variété de tout ce qu'on peut faire finalement en linguistique (...) Cela finira malgré moi par un livre où, sans enthousiasme, j'expliquerai pourquoi il n'y a pas un seul terme employé en linguistique auquel j'accorde un sens quelconque. Et ce n'est qu'après cela, je l'avoue, que je pourrai reprendre mon travail au point où je l'avais laissé" (lettre à Antoine Meillet, 4/1/1894).

Pendant deux décennies, Saussure n'a cessé d'élaborer une linguistique générale, en essayant de répondre à trois questions fondamentales:

(1) Quel type de science la linguistique est-elle?

(2) Quel est son objet?

(3) Quelles sont les manifestations de cet objet?

\footnotetext{
${ }^{5}$ Cf. Saussure (1916: 13-16): "La science qui s'est constituée autour des faits de langue a passé par trois phases successives avant de reconnaitre quel est son véritable et unique objet. On a commencé par faire ce qu'on appelait de la 'grammaire' (...) Ensuite parut la philologie (...) La troisième période commença lorsqu'on découvrit qu'on pouvait comparer les langues entre elles. Ce fut l'origine de la philologie comparative ou 'grammaire comparée' (...) Mais cette école, qui a eu le mérite incontestable d'ouvrir un champ fécond, n'est pas parvenue à constituer la véritable science linguistique. Elle ne s'est jamais préoccupée de dégager la nature de son objet d'étude. Or, sans cette opération élémentaire, une science est incapable de se faire une méthode".
} 
La première question reflétait les préoccupations de l'épistémologie de l'époque: il s'agissait de justifier une classification logique des sciences, et à cet égard la place de la linguistique - science théorique ou science historique - était loin d'être claire. Saussure adopta une position ferme à ce propos: pour lui, la linguistique est une science nomologique, et plus particulièrement sémiologique. Son objet est constitué par les lois qui président à la formation (et à l'évolution) de systèmes de signes, situés dans le temps.

"On a discuté pour savoir si la linguistique appartenait à l'ordre des sciences naturelles ou des sciences historiques. Elle n'appartient à aucun des deux, mais à un compartiment des sciences <qui, s'il n'existe pas, devrait exister sous le〉 nom de sémiologie, c'est-à-dire science des signes ou étude de ce qui se produit lorsque l'homme essaie de signifier sa pensée au moyen d'une convention nécessaire. <Parmi tous les systèmes sémiologiques> le système sémiologique 'langue' est le seul (avec l'écriture) qui ait eu à <affronter cette épreuve [de] se> trouver en présence du Temps, qui ne se soit pas simplement <fondé> de voisin à voisin par mutuel consentement, mais aussi de père en fils par impérative tradition et au hasard de ce qui arriverait en cette tradition, chose hors de cela inexpérimentée <non connue ni décrite>. Si l'on veut, la linguistique est donc une science psychologique en tant que sémiologique, mais les psychologues n'ont jamais fait intervenir le TEMPS dans leur sémiologie. Ce fait qui est le premier qui puisse exciter l'intérêt $<\mathrm{du}>$ philosophe reste ignoré des philosophes; aucun d'eux n'enseigne ce qui se passe dans la transmission d'une sémiologie. Et ce <même> fait accapare en revanche tellement l'attention des linguistes que ceux-ci en sont à croire <pour cela> que leur science est historique ou éminemment historique, n'étant rien d'autre que sémiologique: par là complètement comprise d'avance dans la psychologie, 〈à> condition que celle-ci voie de son côté qu'elle a dans la langue un objet s'étendant à travers le temps, et la forçant de sortir absolument de ses spéculations sur le signe momentané et l'idée momentanée" (Ms. BPU Genève, Ms. fr. 3951, Note 24) ${ }^{6}$.

C'est de la considération de l'objet qu'est le signe ${ }^{7}$, unité biface, que suivent les dichotomies saussuriennes:

(a) celle entre la matière du signe et sa valeur: le signe se définit non pas par sa substance, mais par ses oppositions avec d'autres signes;

(b) celle entre langue et parole: la langue est un héritage continu, alors que la parole en est l'exploitation concrète et discontinue. Dans la langue, le "signe est immuable";

(c) celle entre synchronie et diachronie: la synchronie est un état où il y a des valeurs, c'est-à-dire où les unités du système sont définies par des oppositions,

\footnotetext{
6 À propos de ce texte (manuscrit) de Saussure et son importance pour une analyse épistémologique du projet saussurien, voir Swiggers (2000, 2001b).

${ }^{7}$ En ce qui concerne l'élaboration d'une théorie linguistique basée sur le concept de "signe", voir Swiggers $(1996,2000)$
} 
comme dans n'importe quel système d'économie. C'est la parole qui fait évoluer la langue vers une autre synchronie: évolution qui peut changer ou déplacer le rapport entre tel signifiant et tel signifié. Mais l'évolution même, l'objet de la diachronie, n'est pas un système: le fait diachronique n'a pas de valeur en lui-même, il ne s'oppose à aucun autre fait;

(d) celle entre rapports associatifs et rapports syntagmatiques: les rapports associatifs sont des rapports reliant des signes selon leur constitution en signifiant et signifié, dans la langue intériorisée, alors que le siège des groupes syntagmatiques est dans la parole extériorisée, vu qu'il s'agit de termes présents dans une série effective.

C'est par rapport à cette tentative d'élaboration d'une science linguistique par Saussure qu'il faut comprendre le projet de Hjelmslev. Celui-ci est plus englobant et plus précis: plus englobant, parce qu'il définit le champ d'une sémiotique qui comprend 'langage' et 'non-langage', et plus précis, parce qu'il indique les propriétés distinctives de l'objet (ou des objets) qu'on étudie dans la théorie du langage.

$\mathrm{La}$ voie qu'a prise Hjelmslev, dans ses premiers travaux, a été celle de définir la langue comme une forme ${ }^{8}$. Nous nous proposons d'étudier ici en détail sa démarche initiale, formulée dans les Principes de grammaire générale (1928), où Hjelmslev a voulu poser les bases d'une science des catégories linguistiques. Au centre de ce travail est l'idée que la langue est une "forme qui s'exprime dans une série de catégories constituant un système" (1928: 7). Le projet d'une grammaire scientifique dépend crucialement de deux exigences:

(a) celle d'adopter un point de vue strictement synchronique

(b) celle de se baser sur la forme linguistique, dans son rapport avec une fonction.

La première exigence, pour laquelle Hjelmslev s'inspire de la dichotomie saussurienne - tout en faisant remarquer qu'elle apparaît déjà chez le grammairien danois H.G. Wiwel - consiste à affirmer que le système ne peut être envisagé que d'un point de vue synchronique. C'est que l'axe synchronique recoupe la structure de la langue en tant que système analysable ou décomposable. Hjelmslev a bien vu que les deux axes que Saussure a construits pour représenter l'état et le déroulement, en tant que perspectives d'approche, doivent se combiner avec ce que les deux axes permettent d'aborder au niveau de l'objet: la première subdivision de la linguistique - en linguistique synchronique et diachronique - se croise donc avec une autre subdivision, celle en théorie des sons, théorie des formes, théorie des mots et théorie des syntagmes (cf. Hjelmslev 1928: 48). Hjelmslev observe à ce propos que

8 Cf. aussi Hjelmslev - Uldall (1936: 1): "As particularly emphasised by F. de Saussure, language is form, not substance. Language is the form through which the physical substance and the substance of ideas, which are in themselves amorphous, are made to correspond to each other as expression and content respectively. Consequently, all linguistic analysis must be morphological and cannot be achieved through a mere description of the substances. As the linguistic form does not necessarily coincide with any other known form, this morphology must be immanent". 
si la théorie des sons est en équilibre entre les deux axes, on passe vers l'étude synchronique en s'acheminant vers les mots et, surtout, vers les syntagmes. La synchronie s'explique par elle-même, à l'inverse de la diachronie (qui, en fait, n'est qu'une juxtaposition d'états synchroniques).

La deuxième exigence - à savoir qu'il faut partir de la forme, dans son rapport avec une fonction - implique deux choses:

(a) la grammaire comprend l'étude des sémantèmes et des morphèmes, et de leur combinaison (ce qui constitue d'ailleurs la fonction) ${ }^{9}$;

(b) la morphologie ne peut être dissociée de la syntaxe.

"Ce qui a été dit pour la syntaxe vaut pour la morphologie également. Les rapports syntagmatiques dominent la morphologie aussi bien que la syntaxe proprement dite. D'un certain point de vue, tous les rapports grammaticaux sont des rapports transitifs. Toute morphologie est syntaxe. Toute syntaxe est morphologie (...)

Étant donné que, en réalité, tout fait syntaxique est morphologique en ce sens qu'il concerne uniquement la forme grammaticale, et étant donné également que tout fait morphologique peut être considéré comme syntaxique puisqu'il repose toujours sur une connexion syntagmatique entre les éléments grammaticaux en question, nous sommes persuadé que la division possible de la grammaire en morphologie et syntaxe est dénuée de toute importance du point de vue pratique. La grammaire est, en effet, une discipline une, la théorie de la forme tout court. Elle est entièrement différente de la théorie des sons. C'est cette division seule qui importe, et nullement cette autre entre morphologie et syntaxe" (1928: 53, 94).

L'analyse d'un système doit se faire donc au plan synchronique; mais il ne s'agit là que d'une étude de grammaire immanente, et concrète. Or, Saussure avait utilisé le terme de synchronie dans un autre sens aussi, lorsqu'il écrivait: “L'objet de la linguistique synchronique générale est d'établir les principes fondamentaux de tout système idiosynchronique, les facteurs constitutifs de tout état de langue ... C'est à la synchronie qu'appartient tout ce qu'on appelle la "grammaire générale»" (Saussure 1916: 141). Hjelmslev propose de réserver le terme de "panchronique" à la perspective qui consiste à intégrer dans un état abstrait les faits observés dans l'étude synchronique des langues ${ }^{10}$. Il rejoint sur ce point la conception de Meillet à propos de la linguistique générale (que Meillet voit surtout comme une théorisation de la linguistique diachronique): la linguistique générale

${ }^{9}$ Cf. Hjelmslev (1928: 123): "Nous comprenons par fonction grammaticale: $1^{\circ}$ la faculté de se combiner exclusivement avec certains morphèmes donnés, et $2^{\circ}$ la faculté de se combiner avec les autres sémantèmes exclusivement au moyen de certains morphèmes donnés".

10 "Sous cette réserve et dans ce sens spécial, nous pouvons très bien désigner la grammaire scientifique comme une discipline panchronique. La mise au point des possibilités panchroniques sera - nous le croyons fermement - le but de toute linguistique générale. C'est donc en ce sens aussi que la grammaire doit travailler" (Hjelmslev 1928: 104). Voir aussi le passage suivant: "on ne peut pas repousser ou abolir la notion des catégories, précisément parce qu'elles sont une qualité fixe et essentielle de l'objet avec lequel on travaille" (Hjelmsiev 1928: 83). 
est l'étude des possibilités générales, dont certaines sont exploitées, dans diverses orientations, par différentes langues ${ }^{11}$. C'est à l'élaboration d'une telle linguistique panchronique - et plus particulièrement à une grammaire panchronique, prenant comme objet la forme grammaticale - que Hjelmslev se consacre dans les Principes de grammaire générale.

"Enfin, la grammaire synchronique, en tant qu'elle existe, ne s'est pas placée sur le terrain panchronique. Une grammaire générale, et en même temps, d'ordre linguistique et synchronique, n'existe pas encore. La grammaire synchronique n'a donc pas pu énoncer des règles, n'a pas pu formuler les possibilités générales et les conditions nécessaires qui régissent le mécanisme du langage. Elle n'a pas pu donner les directives nécessaires pour l'étude diachronique et dont celle-ci a si grandement besoin" (Hjelmslev 1928: 109).

L'objet de la grammaire générale, c'est la forme grammaticale, que Hjelmslev qualifie d'abstraite et d'algébrique, et qu'il divise en sémantèmes, en morphèmes et en fonctions ${ }^{12}$. C'est dire que la forme, ce sont les catégories grammaticales, abstraction faite de la technique au moyen de laquelle elles s'expriment ${ }^{13}$. La forme grammaticale est toujours située du côté du signifiant:

"On comprendra donc par forme: tout ce qui, dans le signe, est directement tangible à l'exclusion de tout ce qui y est conventionnel. Étant donné que les phonèmes sont de pures conventions, ils n'entrent pas dans la forme. Ce fait n'empêche pas que la forme elle-même soit directement tangible. La forme n'est constituée que par une série de catégories subconscientes, et ces catégories se trouvent uniquement et immédiatement dans l'image verbale; le signifié même, au contraire, ne s'y trouve pas immédiatement- sauf dans le cas où il se revêt d'une forme. La forme fait donc partie du signifiant, et non du signifié" (1928: 116).

Il importe d'observer ici que le concept de 'forme' chez Hjelmslev est un concept fonctionnel: cela ne ressort pas seulement d'affirmations comme celle que "la forme est, pour ainsi dire, un intermédiaire entre la pensée et la parole" (Hjelmslev 1928: 120 ), ou de son analyse (1928: 122) de sheep comme singulier et pluriel à la fois (où on voit que la forme s'identifie avec les possibilités de construction), mais cela appa-

11 Cf. Meillet (1921: 11, 15-16): “On déterminera ainsi, non plus des lois historiques, telles que sont les 'lois phonétiques' ou les formules analogiques qui emplissent les manuels actuels de linguistique, mais des lois générales qui ne valent pas pour un seul moment du développement d'une langue, qui au contraire sont de tous les temps; qui ne sont pas limitées à une langue donnée, qui au contraire s'étendent également à toutes les langues. Et qu'on le remarque, ce ne seront ni des lois physiologiques, ni des lois psychiques, mais des lois linguistiques (...) Les lois de la phonétique ou de la morphologie générale historique ne suffisent donc à expliquer aucun fait; elles énoncent des conditions constantes qui règlent le développement des faits linguistiques". Sur la conception de la linguistique générale chez Meillet, voir Swiggers $(1985,1988,2001 a)$.

12 Cf. Hjelmslev (1928: 161-173), section cruciale consacrée au problème des "catégories grammaticales".

13 "La grammaire, qui n'est que la science de ces catégories, ne peut se constituer sans rendre compte de cette distinction. Cette science a pour objet les catégories grammaticales en elles-mêmes, non la technique au moyen de laquelle elles s'expriment. Les catégories grammaticales, voilà la forme; la technique, voilà l'aspect" (Hjelmslev 1928: 113). 
raît aussi du fait que Hjelmslev a reconnu que la notion de 'function' chez Sapir ${ }^{14}$ correspond à son emploi de 'forme' (Hjelmslev 1928: 117).

La grammaire générale, en tant que science de catégories, est l'étude de formes. Celles-ci se manifestent de trois manières: en tant que sémantèmes, en tant que morphèmes, en tant que fonctions. Les catégories de sémantèmes sont des catégories significatives, marquées dans la forme ${ }^{15}$ : comme exemple, on pourrait penser aux idéophones dans certaines langues africaines ${ }^{16}$. Les catégories de morphèmes sont les marques incidentes aux concepts radicaux: il s'agit des catégories du temps, de la personne, du cas, du nombre et du mode ${ }^{17}$. Les catégories fonctionnelles, enfin, correspondent aux possibilités de relations d'une notion donnée. Hjelmslev les identifie avec les parties du discours ${ }^{18}$; on pourrait préciser qu'il s'agit de l'aspect de consignification que véhiculent des classes de sémantèmes. Dans les Principes de grammaire générale, le but de Hjelmslev est d'établir, à partir de la confrontation de systèmes concrets de catégories, un système abstrait de catégories: celui-ci peut être "projeté en retour sur tous les états idiosynchroniques" (1928: 214). Le problème qui se pose à cet égard est le suivant: peuton poser des "systèmes concrets" des langues - c'est-à-dire ce que Humboldt appelait l'innere Form, ou ce que Georg von der Gabelentz appelait Sprachgeist (cf.

${ }^{14}$ Sapir utilise form pour désigner la forme phonique du signifiant (moyen de l'expression d'une catégorie), et function pour désigner la catégorie; voir par exemple Sapir (1921: 61): "Every language possesses one or more formal methods for indicating the relation of a secondary concept to the main concept of the radical element. Some of these grammatical processes, like suffixing, are exceedingly wide-spread; others, like vocalic change, are less common but far from rare; still others, like accent and consonantal change, are somewhat exceptional as functional processes. Not all languages are as irregular as English in the assignment of functions to its stock of grammatical processes. As a rule, such basic concepts as those of plurality and time are rendered by means of other method alone, but the rule has so many exceptions that we cannot safely lay it down as a principle. Wherever we go we are impressed by the fact that pattern is one thing, the utilization of pattern quite another. A few further examples of the multiple expression of identical functions in other languages than English may help us to make still more vivid this idea of the relative independence of form and function" (cf. Swiggers 1993). On comparera ce passage avec ce que Hjelmslev (1928: 113-114) dit à propos de la multiplicité des aspects (ou techniques) sous lesquels s'exprime une catégorie. Hjelmslev se distancie ici nettement de Sechehaye (1908: 110-114) et de Bally (1922: 125).

15 "Sans perdre de vue la nature arbitraire du signe sémiologique en général et du signe linguistique en particulier, on peut, sous cette réserve, aborder un travail pour dégager des catégories de sémantèmes. Il y a deux domaines où les efforts à cet égard risquent sans doute le moins d'échouer, et où même la possibilité d'un 'symbolisme' est généralement admise: nous voulons parler des sémantèmes imitatifs (ou onomatopées) d'une part, des sémantèmes expressifs, de l'autre" (Hjelmslev 1928: 176-177).

16 Voir, pour d'intéressants exemples, Fivaz (1963) et Fortune (1962). Hjelmslev cite comme exemples les "gestes vocaux" en éhoué, en ronga et en chinook.

${ }^{17}$ Selon Hjelmslev, qui en fournit un traitement extrêmement sommaire, "le contenu significatif de ces catégories n'est pas moins évident que leur existence même" (Hjelmslev 1928: 197).

18 "Il est donc évidemment possible d'établir des catégories constituées par les sémantèmes qui ont une même fonction. Tous les sémantèmes n'ont pas la même fonction, mais il existe des catégories de sémantèmes dont la fonction est identique. Ce sont là les catégories fonctionnelles. Les catégories fonctionnelles sont identiques à ce qu'on appelle généralement les parties du discours" (Hjelmslev 1928: 199). 
aussi Boas et Sapir $)^{19}$ - à un niveau abstrait, formant un système ? Hjelmslev s'oppose ici au relativisme de Lévy-Bruhl, selon qui il y aurait un abîme profond qui sépare la mentalité primitive et la mentalité des peuples civilisés (cf. Lévy-Bruhl 1910, 1922). Appliquant cette idée aux catégories linguistiques - qui constituent selon Lévy-Brubl le moyen essentiel de représentation - il faudra conclure qu'une grammaire générale est une chimère ${ }^{20}$. Le fondement ethnologique de la théorie de Lévy-Bruhl a été critiqué par Boas (1911b: 124-154) et par Allier (1927: 66-70, 175-182, 226-236); Hjelmslev, de son côté, ne considère que son application en linguistique (1928: 257-267). Il nous semble que sur ce point Hjelmslev se rattache aux idées de Van Ginneken (1907), de Sechehaye (1926), de Schuchardt (1922: $198)^{21}$ et de Boas (1911a), quatre auteurs qui affirment qu'il y a certains procédés grammaticaux communs à toutes les langues, reflétant l'identité universelle des processus psychologiques fondamentaux. Hjelmslev doit reconnaître toutefois qu'il s'agit là seulement de quelques intuitions à propos de l'Elementare Verwandtschaft des langues, et que "la théorie de cette parenté fondamentale des langues n'a pas encore été faite" (1928: 254).

Dans la dernière partie des Principes, Hjelmslev échafaude une théorie à visée panchronique des catégories grammaticales (1928: 296-340). Cette théorie formule un ensemble de possibilités dont les systèmes idiosynchroniques sont des réalisations particulières. Cela implique que l'établissement d'une catégorie abstraite se fait en tenant compte non pas de son extension, mais de son intension, de son contenu significatif:

"La seule méthode possible sera d'établir une catégorie abstraite correspondante à chaque catégorie concrète, sans tenir compte, au préalable, de son étendue. Il faut par exemple, selon

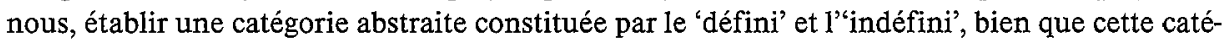
gorie soit inexistante dans un grand nombre d'états concrets. L'existence d'une catégorie dans un seul état concret suffit, en principe, pour conclure que cette même catégorie existe comme possibilité dans les bases psychologiques du langage. La catégorie abstraite n'est rien qu'une possibilité abstraite" (1928: 271).

Hjelmslev veut ainsi construire un système latent de catégories grammaticales, qui permet l'intégration et la comparaison des faits grammaticaux connus. Intégration: cela veut dire qu'on accorde sa vraie valeur systémique à un ensemble de données qu'on observe (comme par ex. les séries de démonstratifs qu'une langue utilise, ou l'ensemble des marques de classe qu'elle utilise); comparaison: cela veut dire qu'on assigne à une donnée grammaticale observée dans telle langue une valeur de "comparat"22 systémique - valeur qui doit être attribuée sans tenir compte de l'étiquetage grammatical de cette donnée dans les grammaires descrip-

\footnotetext{
${ }^{19}$ Voir Humboldt (1883-1884: vol. I, 41-43, 67-70; vol. II, 339-340); von der Gabelentz (1891: 9); Boas (1911a: 81); Sapir (1921: $56,65,115,121,127,134$, qui emploie "inner form" et "genius of a language").

20 Pour ce raisonnement, voir Lévy-Bruhl (1910: 70, 152).

21 Sur l'apport de Schuchardt en linguistique générale, cf. Swiggers (1989).

22 Par ce terme, nous désignons un "fait comparatif/élément de mise en comparaison (épistémologique)"
} 
tives et normatives -, ce qui permettra une comparaison adéquate avec des données d'autres langues.

La grammaire générale telle que Hjelmslev la conçoit doit donc définir, en termes formels, les conditions sous lesquelles des catégories fonctionnelles universelles se réalisent comme des fonctions de sémantèmes (les morphèmes n'ayant pas de fonction dans la théorie de Hjelmslev) dans une hiérarchie de détermination (subordination/rection) et en combinaison éventuelle avec certains marquages morphologiques ${ }^{23}$. Hjelmslev pose ainsi cinq catégories fondamentales ${ }^{24}$ : le substantif, l'adjectif, le pronom, l'adverbe et le verbe.

(1) l'adjectif est un sémantème ayant d'ordinaire fonction de terme secondaire et qui est susceptible de morphèmes de cas dans les états de langue concrets où existent des catégories de cas (cf. Hjelmslev 1928: 308);

(2) le verbe est un sémantème ayant d'ordinaire fonction de terme secondaire et qui n'est pas susceptible de morphèmes de cas dans les états de langue concrets où existent des catégories de cas (cf. Hjelmslev 1928: 308);

(3) le substantif est un sémantème ayant d'ordinaire fonction de terme primaire (cf. Hjelmslev 1928: 306);

(4) l'adverbe est un sémantème ayant d'ordinaire fonction de terme tertiaire (cf. Hjelmslev 1928: 307);

(5) le pronom est une catégorie qui est constituée par les sémantèmes qui sont immobiles à l'égard des catégories de concrétisation: ces sémantèmes restent abstraits dans n'importe quelle combinaison et sans égard à leur emploi (cf. Hjelmslev 1928: 337).

On notera que la cinquième catégorie se soustrait à la définition générale des parties du discours fondamentales, qui est basée sur la notion de subordination. On pourrait se demander alors si la classe des pronoms ne devrait pas être érigée en une catégorie de catégories fonctionnelles: on pourrait y inclure ainsi les classificateurs de langues comme le chinois, et aussi des pro-verbaux comme ceux du basque.

Plus généralement, on pourra se demander si dans les Principes de grammaire générale Hjelmslev ne reste pas trop attaché au système des parties du discours, et si on ne pourrait pas atteindre un niveau plus élevé de généralisation en adoptant comme cadre typologique

(1) la possibilité ou l'impossibilité d'intégrer les concepts relationnels aux concepts concrets; on touche là à la distinction fondamentale entre ce que Sapir (1921: 145-155) appelle "pure-relational languages" et "mixed-relational languages" (cf. Swiggers 1993).

23 Chaque sémantème possède la faculté de se combiner avec les autres sémantèmes de la série, mais seulement au moyen de certains morphèmes donnés. Il est donc possible d'établir des catégories constituées par les sémantèmes qui ont une même fonction: ce sont les "catégories fonctionnelles".

24 "Il s'agit de trouver, pour chacune des catégories fondamentales, la catégorie de morphèmes qui peut fournir un critérium pour la définition de la catégorie fondamentale en tant que telle" (Hjelmslev 1928: 306). 
(2) des rapports morpho-syntaxiques qui ne se manifestent pas seulement au niveau morphologique (comme dans les langues à cas, ou comme dans les langues à "états nominaux" - état absolu, état construit, état défini - , telles que les langues sémitiques), au niveau syntaxique (comme par exemple en chinois), ou au niveau tonologique (cf. les langues bantoues): je pense ici à des complexes de rapports comme "déterminant - déterminé - ni déterminant, ni déterminé", "noyau et périphérie", "base et adjonction".

Une telle analyse permettrait de rendre compte des similarités qui se superposent aux différentes parties du discours.

Un défaut plus général des Principes est que Hjelmslev y élabore une théorie du système morphologique du langage sans poser les bases d'une théorie des morphèmes - ce que l'auteur admet d'ailleurs:

"Il y a un problème particulier qu'il est évidemment nécessaire de trancher avant de pouvoir engager ces études. C'est le problème de la nature du morphème. Nous avons effleuré ce problème à plusieurs reprises au cours de ce livre. En effet, c'est là un des problèmes les plus fondamentaux de la science grammaticale. On ne peut pas envisager une solution des problèmes plus spéciaux sans savoir d'avance ce qui, dans un état de langue, est à considérer comme un morphème et ce qui est à considérer comme un sémantème" (1928: 339).

En guise de conclusion à cette étude d'historiographie de la linguistique générale, que nous prenons plaisir à dédier à notre collègue Janez Orešnik, essayons de déterminer le statut épistémologique de l'entreprise hjelmslévienne ${ }^{25}$. On peut la caractériser comme une réflexion sur "l'organisation" du langage, ce qui implique une caractérisation des types linguistiques et une définition des catégories linguistiques. Ce type de réflexion rattache l'œuvre de Hjelmslev à celle de Saussure, de Sapir et de Jespersen (cf. Swiggers 1991). Cette réflexion comprend deux volets grammaticaux importants: (1) l'analyse en catégories générales de description grammaticale (on peut penser ici à des catégories comme hiérarchie, dépendance, intégration, variation, appartenance à une classe, etc.; (2) le second volet est celui de la réflexion sur les propriétés de la description grammaticale - propriétés abstraites (cf. dimensionnalité, simplicité, caractère multifactoriel, non-contradiction) et propriétés empiriques (adéquation descriptive, exhaustivité matérielle, ...). Hjelmslev a posé ainsi, en 1928 , les bases de sa théorie de linguistique générale, qui répond à une double tâche: celle d'analyser la stratification intérieure du langage, comme "genre" par rapport aux "espèces"-langues, et celle de situer cette structuration comme objet et pratique sémiotiques dans une théorie de la construction du sens.

$\overline{25}$ Voir aussi, pour des éléments de comparaison avec la linguistique américaine, Swiggers $(1991,1996)$. 


\section{Références bibliographiques}

ALBRECHT, Jörn. 1988. Europäischer Strukturalismus: Ein forschungsgeschichtlicher Überblick. Tübingen: Francke. ALLIER, Raoul. 1927. Le non-civilisé et nous: différence irréductible ou identité foncière ? Paris: Payot.

BaLly, Charles. 1922. "La pensée et la langue". Bulletin de la Société de Linguistique de Paris 23. 117-137.

BOAS, Franz. 1911a. "Introduction". Handbook of American Indian Languages vol. I, 5-83. Washington: Bureau of American Ethnology.

BoAs, Franz. 1911b. The Mind of Primitive Man. New York: Macmillan.

FISCHER-JøRGENSEN, Eli. 1943. compte rendu de Hjelmslev 1943. Nordisk Tidsskrift for Tale og Stemme 7. 81-96.

Frvaz, Derek. 1963. Some Aspects of the Ideophone in Zulu. Hartford: Seminary Foundation. (Thèse).

FORTUNE, G. 1962. Ideophones in Shona. Oxford: University Press.

GABELENTZ, Georg VON DER. 1891. Die Sprachwissenschaft: ihre Aufgaben, Methoden und bisherigen Ergebnisse. Leipzig: Weigel. [1901²]

HJeLMSLEV, Louis. 1928. Principes de grammaire générale. (Det Kgl. Danske Videnskabernes Selskab. Historiskfilologiske Meddelelser XVI, 1). Copenhagen: A.F. Høst \& Søn.

HJELMSLEV, Louis. 1943. Omkring Sprogteoriens Grundlceggelse. [Festskrift udgivet af Kobenhavns Universitet, 1113]. Copenhagen: E. Munksgaard. [Réimpressions: Akademisk Forlag, 1966; Travaux du Cercle Linguistique de Copenhague, vol. XXV, 1993]. [Traduction française: voir sous HJELMSLEV 1968]

HJELMSLev, Louis. 1968. Prolégomènes à une théorie du langage. Paris: Éditions de Minuit. [Nouvelle édition: par Una Canger et Annick Wewer, Paris: Éditions de Minuit, 1971]

HJelmSLeV, Louis - UldDall, Hans J. 1936. Synopsis of an Outline of Glossematics. [Pamphlet]

HUMBOLDT, Wilhelm vON. 1883-1884. Die sprachphilosophischen Werke. Herausgegeben von H. Steinthal. Berlin: Dümmler. [2 vol.]

LÉVY-BRUHL, Lucien. 1910. Les fonctions mentales dans les sociétés inférieures. Paris: Alcan.

LEVY-BruHL, Lucien. 1922. La mentalité primitive. Paris: Alcan.

MEILLET, Antoine. 1921. Linguistique historique et linguistique générale (t. I). Paris: Champion.

SAPIR, Edward. 1921. Language. An introduction to the study of speech. New York: Harcourt, Brace and Company.

SAUSSURE, Ferdinand DE. 1916. Cours de linguistique générale. Édité par Charles Bally, Albert Sechehaye et Albert Riedlinger. Paris: Payot.

SCHUCHARDT, Hugo. 1922. Schuchardt-Brevier. Ein Vademecum der allgemeinen Sprachwissenschaft. Eingeleitet von Leo Spitzer. Halle: Niemeyer.

SeChenaYk, Albert. 1908. Programme et méthodes de la linguistique théorique. Paris: Champion; Leipzig: Harrassowitz.

SECHEHAYE, Albert. 1926. Essai sur la structure logique de la phrase. Paris: Champion.

SwIGGERS, Pierre. 1985. "La linguistique historico-comparative d'Antoine Meillet: Théorie et méthode". Cahiers Ferdinand de Saussure 39. 181-195.

SwiGgers, Pierre. 1988. "Le problème du changement linguistique dans l'œuvre d'Antoine Meillet". Histoire, Épistémologie, Langage 10:2. 155-166.

SwiGgers, Pierre. 1989. "Linguistique historique, générale et particulière chez Hugo Schuchardt". Folia Linguistica Historica 8. 219-231.

SwIGGERs, Pierre. 1991. "Note sur la linguistique générale en 1921-1922. Avec l'édition de deux lettres de Joseph Vendryes à Edward Sapir”. Beiträge zur Geschichte der Sprachwissenschaft 1. 185-191.

SwIGGERs, Pierre. 1995. "Le programme d'une linguistique générale chez Louis Hjelmslev". Bulletin de la Société de Linguistique de Paris 90. 53-83.

Swiggers, Pierre. 1993. "'Synchrony' and 'Diachrony' in Sapir's Language (1921)”. Neuphilologische Mitteilungen 94. 313-322.

SwIGGERs, Pierre. 1996. "XX-th Century Theories of Language: An Epistemological Diagnosis". Linguistica 36. 3-16.

SwIGGers, Pierre. 2000. "Linguistic Sign". In: Morphology. An International Handbook on Inflection and WordFormation, vol. I, 210-224. Berlin - New York: W. de Gruyter.

SwIGGERs, Pierre. 2001a. "Antoine Meillet et la notion de «linguistique générale»". In: Anne-Marie LofflerLaurian (éd.), Études de linguistique générale et contrastive. Hommage à Jean Perrot, 413-426. Paris: Institut d'études hongroises.

SwIGGERs, Pierre. 2001b. "«Comme un canard couvé par une poule»: Saussure devant l'objet de la linguistique (générale)". Incontri linguistici 24. 11-21.

VAN GINNEKEN, Jacques. 1907. Principes de linguistique psychologique: essai de synthèse. Paris: Rivière. [2 vol.] 


\section{Povzetek \\ OBLIKE, FUNKCIJE IN KATEGORIJE V JEZIKU: PRINCIPES DE GRAMMAIRE GÉNÉRALE LOUISA HJELMSLEVA}

V svojem delu Principes de grammaire générale (1928) je Louis Hjelmslev zasnoval teoretične temelje morfološkega sistema v jeziku, pri čemer je osrednji pojem 'oblika'. Vendar pa ne najdemo celostne teorije, ki bi dovoljevala členiti razporeditev kategorij (semantemov, morfemov in funkcij): V svojih Principih Hjelmslevu ni uspelo izdelati dokončno teorijo za zgradbo morfematike. To izvira iz dejstva, da koncept "oblike" za Hjelmsleva ni zaključek uporabe analitičnih postopkov, ampak predstavlja soodnosni člen tistega, kar je poistovetil s "funkcijo". Pri Hjelmslevu slovnične kategorije niso definirane $\mathrm{z}$ mejami ekstenzivnosti, ampak z mejami pomenske vsebine. A že $\mathrm{v}$ tem delu iz leta 1928 je vidna osnovna konstanta raziskav: raziskava abstraktnega sistema, ki dovoljuje definirati vse enote in pojasniti vse možne postopke, obenem pa uporabiti deđuktivno metodo, torej sklepanje od splošnega $\mathrm{k}$ posameznemu, vse to $\mathrm{z}$ namenom, da bi bilo mogoče izdelati teorijo jezikovne strukture. 